\title{
CHLAMYDONEMA MASOODI N. SP. (1)
}

Par M. B. MIRZA (2)

Deux chats sauvages (Felis chaus) ont été disséqués au laboratoire de zoologie par MM. M.-H. Burney et Satya Narain Singh. Les parasites m'ont été remis, pour identification, dans deux tubes séparés.

Le tube $\mathrm{n}^{\circ} 1$ renfermait 19 exemplaires de Chlamydonema, dont 3 mâles, 6 femelles et 10 larves. La longueur des mâles variait de 18 à $33 \mathrm{~mm}$. pour un diamètre de $0 \mathrm{~mm}$., 9 à $2 \mathrm{~mm}$., 3. Les femelles mesuraient de 19 à $28 \mathrm{~mm}$. de longueur, pour un diamètre de $0 \mathrm{~mm} .9$ à $2 \mathrm{~mm}$., 2. Quatre spécimens adultes seulement ont été trouvés dans l'estomac : tous les autres provenaient de l'intestin grêle.

Le tube $\mathrm{n}^{\circ} 2$ renfermait 500 exemplaires, dont 477 étaient des larves de Chlamydonema, toutes provenant de l'intestin grêle. Il n'y avait que 23 spécimens adultes dont seulement 6 mâles, le reste étant des femelles; quelques-uns avaient été trouvés dans l'estomac et un petit nombre dans l'intestin grêle. On peut en conclure que les larves arrivent à maturité dans l'intestin grêle et émigrent ensuite dans l'estomac. Les mâles mesuraient de 19 à $26 \mathrm{~mm}$. de longueur, sur un diamètre de $0 \mathrm{~mm}$., 9 à $1 \mathrm{~mm}$., 8 . La longueur des femelles variait de 19 à $29 \mathrm{~mm}$., pour un diamètre de $0 \mathrm{~mm}$., 9 à $2 \mathrm{~mm} ., 2$.

Le corps est atténué en avant et l'extrémité postérieure, chez tous les exemplaires, à l'exception des larves, est enveloppée par une cuticule très lâche qui s'étend bien au-delà de cette extrémité. La longueur de ce repli cuticulaire, à partir de la pointe de l'extrémité postérieure, mesure $1 \mathrm{~mm}$., 21 chez les mâles et $3 \mathrm{~mm}$., 09 chez les femelles. La cuticule est striée transversalement et est seulement légèrement réfléchie sur les lèvres.

Les lèvres sont grandes et coniques; chacune d'elles porte une grande dent médiane externe, obtusément triangulaire, et une dent médiane interne tripartite. Chaque lèvre possède, en outre, deux papilles externes très visibles, l'une sub-dorsale et l'autre sub-ventrale. A la base de chaque lèvre, il y a deux protubérances coniques

(1) Dédié au Dr. Sir S.R. Masood, Vice-Chancelier, Muslim University, Aligarh, U.P. India.

(2) Traduit de l'anglais par le Dr. Maurice Langeron.

Annales de Parisitologie, t. XII, $\mathbf{x}^{\circ}$ 5. $-1^{\text {er }}$ septembre 1934, p. 367-370. 
proéminentes. Le pore excréteur est situé à $0 \mathrm{~mm}$. 92 de l'extrémité antérieure.

L'œsophage est cylindrique et plus large au voisinage de sa partie postérieure. Sa longueur est de $4 \mathrm{~mm}$., $62 \mathrm{chez}$ une femelle de $23 \mathrm{~mm}$.

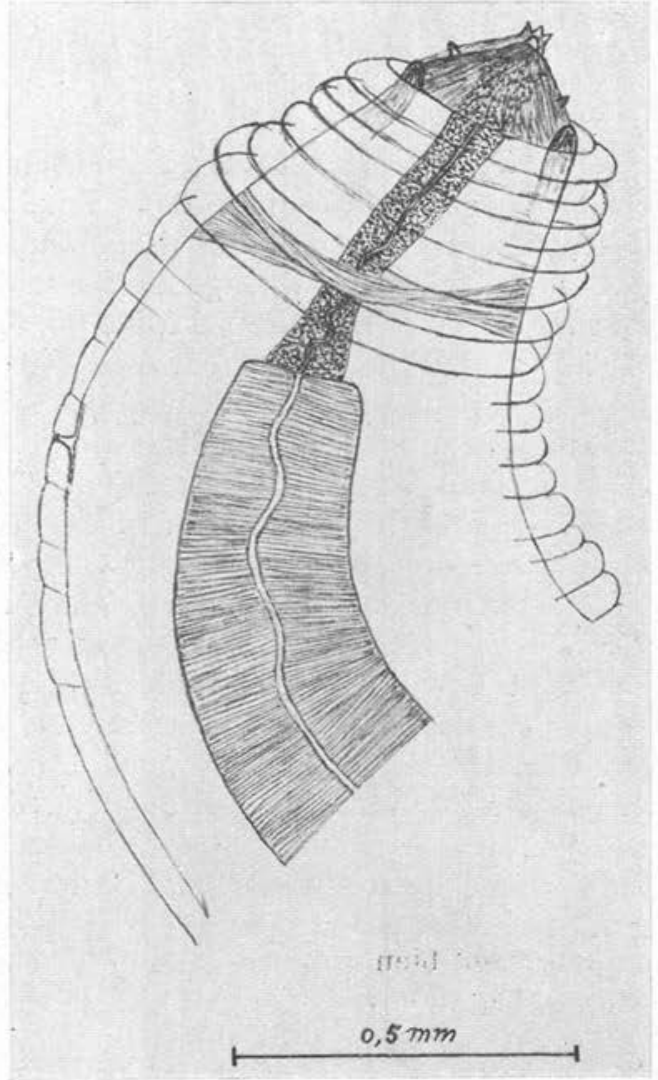

Fig. 1. - Chlamydonema masoodi n. sp. - Vue latérale de la tête, montrant les lèvres, les papilles, l'anneau nerveux et le pore excréteur.

de longueur. Il n'y a pas de cavité buccale entre les lèvres et l'œsophage. L'ameau nerveux est grand et entoure l'œsophage dans son quart postérieur.

Chez toutes les femelles adultes, un anneau chitineux détachable entoure le corps dans la région de la vulve. Celle-ci se trouve à $10 \mathrm{~mm}$., 1 de l'extrémité antérieure d'une femelle mesurant $23 \mathrm{~mm}$. 
La queue est pointuè et légèrement recourbée ventralement. Les organes génitaux ressemblent à ceux du Chlamydonema præputialis et sont du groupe didelphys.

Chez les mâles, l'extrémité postérieure est obtusément pointue

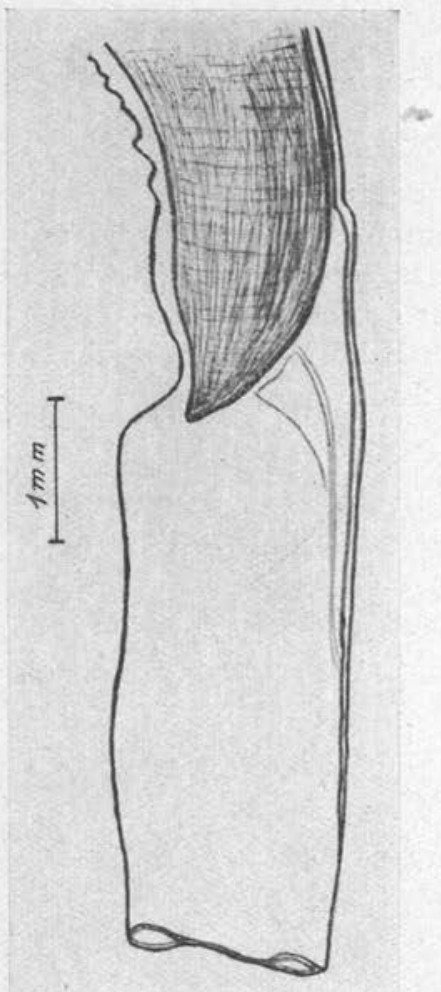

F IG. 2. - Chlamydonema masoodi n. sp. - Vue latérale de l'extrémité postérieure de la femelle, montrant le repli cuticulaire.

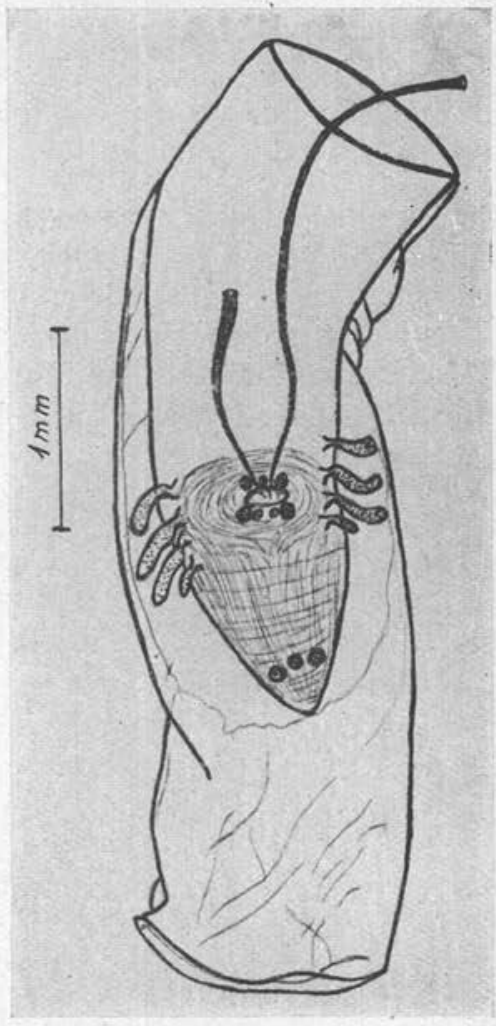

Fig. 3. - Chlamydonema masoodi $\mathrm{n}$ sp. - Face ventrale de l'extrémité postérieure du mále, montrant les spicules, les papilles anales et le repli cuticulaire.

et recourbée ventralement. Il y a quatre paires de papilles pédiculées péricloacales, deux paires de papilles préanales et deux paires de papilles post-anales. Celles-ci sont globuleuses au milieu mais obtusément pointues à la partie externe. Les trois papilles préanales ventrales sont disposées en une rangée. Celle du milieu est plus petite que les deux externes. Exactement en arrière de l'anus, il y a 
deux autres paires disposées en une rangée transversale. Les deux papilles externes sont plus grandes que les deux internes. Plus bas, sur la queue, il y a encore trois papilles sur une rangée.

Les spicules sont inégaux, courbes et pointus. Le spicule gauche est long et mince, tandis que le droit est plus court et plus épais. Les spicules n'ont été mesurés que dans deux cas et les dimensions trouvées ont été les suivantes :

$\begin{array}{lll}\text { Spicule droit : } & \text { a) } 1 \mathrm{~mm} ., 31, b) & 0 \mathrm{~mm} ., 82 \\ \text { Spicule gauche : } & \text { a) } 2 \mathrm{~mm} ., 52, b) & 1 \mathrm{~mm} ., 28\end{array}$

Cette espèce semble, sous certains aspects, identique à Chlamydonema prxputialis et $C$. malayensis ; toutefois, on ne peut la confondre avec ces espèces, car elle en diffère nettement par le nombre, les dimensions et la disposition des papilles anales ventrales.

Les types sont déposés au Musée du Laboratoire de Zoologie de la Muslim University, à Aligarh, U.P., Inde.

Laboratoire de Zoologie, Muslim University, Aligarh, U.P. Inde. 